\title{
JOINS OF TOPOLOGIES HOMEOMORPHIC TO THE RATIONALS
}

\author{
A. J. JAYANTHAN AND V. KANNAN
}

(Received 9 November 1987; revised 6 June 1988)

Communicated by J. H. Rubinstein

\begin{abstract}
Let $Q$ be the space of all rational numbers and $(X, \tau)$ be a topological space where $X$ is countably infinite. Here we prove that (1) $\tau$ is the join of two topologies on $X$ both homeomorphic to $\mathbf{Q}$ if and only if $\tau$ is non-compact and metrizable, and (2) $\tau$ is the join of topologies on $X$ each homeomorphic to $Q$ if and only if $\tau$ is Tychonoff and noncompact.
\end{abstract}

1980 Mathematics subject classification (Amer. Math. Soc.) (1985 Revision): 54 E 35.

Keywords and phrases: metric spaces, joins of topologies, countable spaces.

The set of all rational numbers with the usual topology is denoted by $Q$ and is called simply 'rationals.' A topology $\tau$ on a countable set $X$ is called a rational topology if $(X, \tau)$ is homeomorphic to $\mathbb{Q}$. We recall Sierpinski's Theorem [1]: every countable dense-in-itself metric space is homeomorphic to $\mathbb{Q}$.

By Sierpinski's Theorem, we can conclude that a rational topology is homeomorphic to every coarser metric topology on the same set. Since the join of two rational topologies is regular and second countable, it is metrizable [2] (Urysohn's metrization theorem). Moreover the join is also non-compact. Conversely, here we prove that every non-compact metric topology on a countable set arises in this manner. Further, we study the join of an arbitrary collection of rational topologies also.

The work of the first author was financially supported by the Council of Scientific and Industrial Research, India.

(C) 1989 Australian Mathematical Society $0263-6115 / 89 \$ A 2.00+0.00$ 
Suppose $\tau_{1}$ and $\tau_{2}$ are two topologies on a set $X$. Then the join of $\tau_{1}$ and $\tau_{2}$ is denoted by $\tau_{1} \vee \tau_{2}$ and defined as the topology generated by $\tau_{1} \cup \tau_{2}$.

A subset is called clopen in a topology if it is both closed and open. The set of all natural numbers is denoted by $\mathbb{N}$, and the cardinality of $2^{N}$ by $c$.

\section{Joins of two rational topologies}

THEOREM 1.1. Every non-compact metric topology on a countable set is the join of two rational topologies.

REMARK. Before giving the details of the proof, we outline the main ideas therein. For each clopen subset $K$ whose complement is non-compact, we construct a metric $p$ on $X$ such that

(i) $K$ remains closed in $(X, p)$,

(ii) $p$ induces a coarser topology than $d$,

(iii) $(X, p)$ is dense-in-itself, and

(iv) $p$ and $d$ coincide on $K$.

We divide $X$ into two disjoint non-compact clopen subsets $K$ and $K^{\prime}$ and consider the metrics $p$ and $p^{\prime}$ as above. We can prove that the topology of $(X, d)$ is the join of the topologies of $(X, p)$ and $\left(X, p^{\prime}\right)$. An application of Sierpinski's Theorem completes the proof.

Now we explain the construction of the metric $p$. For each isolated point $a$, we choose a sequence of pairwise disjoint clopen subsets $W_{1}, W_{2}, \ldots, W_{n}, \ldots$ such that the distance between $W_{n}$ and $a$, in the new metric, converges to 0 . This makes the point $a$ a limit point in the new metric. When we do similarly for each isolated point, we take care to see that each clopen subset chosen for an isolated point is disjoint from each of the clopen subsets chosen for any other isolated point. Our proof shows that this construction is possible such that the properties (i) to (iv) are satisfied.

Proof of the Theorem. Let $(X, d)$ be a non-compact countable metric space. Since $(X, d)$ is not compact, there exists an (countable) infinite pairwise disjoint cover $\mathcal{M}$ of nonempty clopen subsets of $X$. Write $\mathcal{M}$ as $U \cup U^{\prime}$ where both $U$ and $U^{\prime}$ are infinite subcollections of $M$ with $U \cap U^{\prime}$ empty. Define $K=\{x \in X \mid x \in U, U \in \mathcal{U}\}$ and $K^{\prime}=\left\{x \in X \mid x \in U, U \in \mathcal{U}^{\prime}\right\}$. Then $K$ and $K^{\prime}$ are disjoint open subsets of $(X, d)$.

Let $A=\left\{a_{1}, a_{2}, \ldots, a_{m}, \ldots\right\}$ be the set of all isolated points of $(X, d)$. This may be infinite or finite or even empty. Our first aim is to construct a metric 
$p$ on $X$ such that

(1) the topology of $p$ is coarser than the topology of $d$,

(2) $(X, p)$ is dense-in-itself, and

(3) a subset of $K$ is closed in $(X, p)$ if and only if it is closed in $(X, d)$.

Condition (2) can be achieved only if we can make every point of $A$ into a limit point in $(X, p)$. This is the main idea of the proof.

Suppose $d$ is bounded by 1 . For convenience, we write $U^{\prime}$ as $U^{\prime}=\left\{G_{m, n} \mid\right.$ $m, n \in \mathbb{N}\}$. First we define another metric $d^{\prime}$ on $X$ as follows:

$$
d^{\prime}(y, x)=d^{\prime}(x, y)= \begin{cases}1 & \text { if } x \text { is in } G_{m, n}, y \text { is in } G_{s, t},(m, n) \neq(s, t), \\ 1 & \text { if } x \text { is in } K, y \text { is in } K^{\prime}, \\ d(x, y) & \text { otherwise. }\end{cases}
$$

Since every $G_{m, n}$ and both $K$ and $K^{\prime}$ are clopen in the topology of $d$, the topology of $d^{\prime}$ is equivalent to that of $d$ on $X$. Further, we note that $d=d^{\prime}$ on $K$ and $d(x, y) \leq d^{\prime}(x, y)$ for every $x, y$ in $X$. Next, we define a real valued function $d_{1}$ on $X \times X$ as follows:

$$
d_{1}(x, y)= \begin{cases}\min \left\{1 / n, d^{\prime}(x, y)\right\}, & \text { if } x=a_{m} \text { for some } m \text { and } y \text { is in } G_{m, n} \\ & \text { for the same } m \text { and for some } n \text { in } \mathrm{N}, \\ d^{\prime}(x, y) & \text { otherwise. }\end{cases}
$$

It is clear that $d_{1}$ is not a metric on $X$ whenever $A$ is non-empty. However $d_{1}$ satisfies the property that $d_{1}(x, y) \leq d^{\prime}(x, y)$ for every $x, y$ in $X$. Once again, $d_{1}, d^{\prime}$ and $d$ coincide on $G_{m, n} \times G_{m, n}$ for every $m, n$ in $\mathrm{N}$ and on $K \times K$. Finally, we define

$$
\begin{aligned}
p(x, y)=\inf \left\{d_{1}\left(x, x_{1}\right)\right. & +d_{1}\left(x_{1}, x_{2}\right)+\cdots+d_{1}\left(x_{n}, y\right), d_{1}\left(y, y_{1}\right) \\
& \left.+d_{1}\left(y_{1}, y_{2}\right)+\cdots+d_{1}\left(y_{m}, x\right) \mid x_{i}, y_{i} \in X, m, n \in \mathbf{N}\right\},
\end{aligned}
$$

for all $x, y \in X$. It is not difficult to note that $p$ is a pseudo-metric on $X$.

Claim. $p$ is a metric on $X$. For that, let $x$ and $y$ be two distinct points in $X$. We show that $p(x, y)>0$.

CASE 1. $x$ and $y$ are in $K$. In fact, we prove that $p$ coincides with $d$ in $K \times K$. For that consider a finite set $\left\{x=x_{0}, x_{1}, \ldots, x_{n}, x_{n+1}=y\right\}$ of points of $X$. If $x_{i}$ is in $K$ for all $i \leq n$, then $d_{1}\left(x, x_{1}\right)+\cdots+d_{1}\left(x_{n}, y\right)=$ $d\left(x, x_{1}\right)+\cdots+d\left(x_{n}, y\right) \geq d(x, y)$. On the other hand, if $x_{i}$ is in $K^{\prime}$ for some $i \leq n$, then there exists a number $r \leq n$ such that $x_{r}$ is in $K^{\prime}$ and $x_{r+1}$ is in $K$, and hence $d_{1}\left(x_{r}, x_{r+1}\right)=d^{\prime}\left(x_{r}, x_{r+1}\right)=1$. Hence, $d_{1}\left(x, x_{1}\right)+d_{1}\left(x_{1}, x_{2}\right)+$ $\cdots+d_{1}\left(x_{n}, y\right) \geq 1 \geq d^{\prime}(x, y)=d(x, y)$. Hence $p(x, y) \geq d(x, y)$ for all $x, y$ in $K$. By definition, $p(x, y) \leq d_{1}(x, y)=d(x, y)$ for al $x, y$ in $K$. Hence $p(x, y)=d(x, y)$ for all $x, y$ in $K$. 
CASE 2. $x$ is in $K^{\prime}$ and $y$ is in $K$. Then $x$ is in $G_{m, n}$ for some $m, n$ in $\mathbb{N}$ and hence $p(x, y) \geq \min \left\{1 / n, d^{\prime}(x, y)\right\}>0$.

CASE 3. $x$ and $y$ are in $K^{\prime}$. Then there exist $m, n, s, t$ in $\mathbb{N}$ such that $x$ is in $G_{m, n}$ and $y$ is in $G_{s, t}$. In this case $p(x, y) \geq \min \left\{1 / n, 1 / t, d^{\prime}(x, y)\right\}>0$.

Hence $p$ is a metric on $X$ as we claimed.

Since $d_{1}(x, y) \leq d^{\prime}(x, y)$, we can conclude that $p(x, y) \leq d^{\prime}(x, y)$ for all $x, y$ in $X$. hence the topology of $p$ is coarser than the topology of $d$. This further implies that the set of all isolated points of $(X, p)$ is contained in $A$. However, if $x_{m, n}$ is a point in $G_{m, n}$, then the sequence $\left\{x_{m, n}\right\}_{n=1}^{\infty}$ converges to $a_{m}$ in the topology of $p$ for all $m$ in N. Hence every point in $A$ is a limit point in $(X, p)$. Therefore $(X, p)$ is dense-in-itself and hence homeomorphic to the rationals.

Now, we claim that $K$ is closed in $(X, p)$. For, if $x$ is a point in $K^{\prime}$, say $x$ belongs to $G_{m, n}$ for some $m, n \in \mathrm{N}$, then $p(x, y) \geq \min \left\{1 / n, d^{\prime}(x, y)\right\}$ for all $y$ belonging to $K$. This shows that no sequence from $K$ can converge to a point in $K^{\prime}$. Hence $K$ remains closed in $(X, p)$. Further, the metric $p$ coincides with $d$ on $K$, shows that a subset of $K$ is closed in $(X, p)$ if and only if it is closed in $(X, d)$.

By a similar argument, interchanging $U$ and $U^{\prime}$, and $K$ and $K^{\prime}$ we can construct a metric $p^{\prime}$ on $X$ such that

(a) the topology of $p^{\prime}$ is coarser than the topology of $d$,

(b) $\left(X, p^{\prime}\right)$ is homeomorphic to $\mathbf{Q}$, and

(c) a subset of $K^{\prime}$ is closed in $(X, d)$ if and only if it is closed in $\left(X, p^{\prime}\right)$.

Finally, we claim that the join of the topologies of $p$ and $p^{\prime}$ is the topology of $d$. Since the topologies of $p$ and $p^{\prime}$ are coarser than the topology of $d$, their join is also coarser. On the other hand if $C$ is closed in $(X, d)$, then $C \cap K$ and $C \cap K^{\prime}$ are closed in $(X, p)$ and $\left(X, p^{\prime}\right)$ respectively. Therefore $C=(C \cap K) \cup\left(C \cap K^{\prime}\right)$ is closed in the join.

This completes the proof of the theorem.

REMARK. The proof given for Theorem 1.1 is independent of the cardinality of $A$. In particular if $A$ is empty, then the metric $p$ will be identical with $d^{\prime}$ on $X$. Also the position of $a_{n}$ is insignificant. It can be in any $G_{s, t}$ or $K$. The cardinalities of the $G_{m, n}$ are also irrelevant as long as they are non-empty.

The converse of Theorem 1.1 is also true. 
Theorem 1.2. Let $(X, \tau)$ be a countable space. Then the following are equivalent:

(1) $(X, \tau)$ is metrizable and not compact;

(2) $\tau$ is the join of two rational topologies;

(3) $\tau$ is the join of finitely many rational topologies;

(4) $\tau$ is the join of countably infinitely many rational topologies.

Proof. (4) $\Rightarrow(1)$. If $\tau$ is the join of countably many rational topologies, then $\tau$ is a regular, second countable Hausdorff topology. Hence $\tau$ is metrizable by Urysohn's metrization theorem. That

$(1) \Rightarrow(2)$ follows from Theorem 1.1 that $(2) \Rightarrow(3)$ and $(3) \Rightarrow(4)$ are obvious.

REMARKS. 1.1. Our result can be reformulated as follows. Let $U$ be the usual topology on $\mathbf{Q}$. For every non-compact metric topology $\tau$ on a countable set $X$, there is a permutation $\sigma$ on $\mathbf{Q}$ such that $(X, \tau)$ is homeomorphic to $(\mathbb{Q}, \mathcal{U} \vee \sigma \mathcal{U})$ where $\sigma \mathcal{U}=\{\sigma U \mid U \in \mathcal{U}\}$.

1.2. On a countable set $X$, if a metric topology $\tau$ is such that every noncompact metric topology on $X$ is the join of two topologies $\tau_{1}$ and $\tau_{2}$ such that both $\left(X, \tau_{1}\right)$ and $\left(X, \tau_{2}\right)$ are homeomorphic to $(X, \tau)$, then $(X, \tau)$ must be homeomorphic to the rationals. In other words rational topology is the unique topology having such a property.

1.3. One can also prove the following. There does not exist a countable Hausdorff space $(X, \tau)$ such that every metric (compact or non-compact) topology on $X$ is the join of two topologies homeomorphic to $(X, \tau)$. This is because any two compact Hausdorff topologies are either identical or uncomparable.

1.4. We remark that a similar result is not true on a set $X$ such that $|X|=\mathrm{c}$ that is, there is no metrizable topology on $X$ such that every non-compact metric topology on $X$ is the join of two topologies $\tau_{1}$ and $\tau_{2}$ on $X$ such that $\left(X, \tau_{1}\right)$ and $\left(X, \tau_{2}\right)$ are homeomorphic to $(X, \tau)$. To see this, suppose, that there exists one such topology $\tau$ on $X$. Then $\tau$ must be second countable as there are second countable topologies on $X$. Hence $\tau_{1} \vee \tau_{2}$ is also second countable whenever $\left(X, \tau_{1}\right)$ and $\left(X, \tau_{2}\right)$ are homeomorphic to $(X, \tau)$. But we know that there are many topologies on $X$ that are not second countable (for example the discrete topology).

\section{Joins of arbitrary collections of rational topologies}

A topology is called zero-dimensional if it has a base of clopen sets. We discuss the joins of arbitrary collections of rational topologies. 
THEOREM 2.1. Let $X$ be a countable set and $\tau$ be a Hausdorff topology on $X$. Then the following are equivalent.

(1) $\tau$ is a non-compact, regular (equivalently, normal or zero-dimensional) topology on $X$.

(2) $\tau$ is the join of a collection of rational topologies on $X$.

Proof. (1) $\Rightarrow(2)$. Since $X$ is countable and hence Lindelöf, $(X, \tau)$ is normal if and only if it is regular. Now, by normality of $X$, for a given point $x$ and a closed set $C$ such that $x \notin C$, there exists a continuous function $f: X \rightarrow[0,1]$ such that $f(x)=0$ and $f(C)=1$. Since $f(X)$ is countable, we can choose $a$ in $(0,1)$ such that $a$ is not in $f(X)$. Then $f^{-1}[0, a)$ is both closed and open in $X$. Hence $(X, \tau)$ is zero-dimensional. If $G$ is any clopen subset in $(X, \tau)$, by a standard procedure we can find a regular Hausdorff topology $\tau_{G}$ on $X$ coarser than $\tau$ such that $\tau_{G}$ is non-compact as well as second countable and $\tau_{G}$ contains $G$ as a clopen subspace. Since such a topology $\tau_{G}$ is metrizable (Urysohn metrization theorem), by Theorem 1.1 there exist topologies $\tau_{G}^{\prime}$ and $\tau_{G}^{\prime \prime}$ on $X$ coarser than $\tau_{G}$ such that both $\left(X, \tau_{G}^{\prime}\right)$ and $\left(X, \tau_{G}^{\prime \prime}\right)$ are homeomorphic to $Q$, and $\tau_{G}$ is the join of $\tau_{G}^{\prime}$ and $\tau_{G}^{\prime \prime}$. Thus $\tau$ is the join of $\left\{\tau_{G}^{\prime} \mid G\right.$ is a clopen subset of $\left.X\right\} \cup\left\{\tau_{G}^{\prime \prime} \mid G\right.$ is a clopen subset of $X\}$. This proves that $(1) \Rightarrow(2)$.

To prove the converse, we observe that every countable compact space is metrizable. Further, there does not exist a rational topology coarser than a compact Hausdorff topology on a countable set. Thus the proof is complete.

Remark 2.1. From Theorem 1.2 and Theorem 2.1, we can conclude that one needs uncountably many rational topologies to generate a nonmetrizable regular topology. It is known that there are ultrafilters on a countable (discrete) space $D$ such that the local weight of the point $\mathcal{F}$ in the space

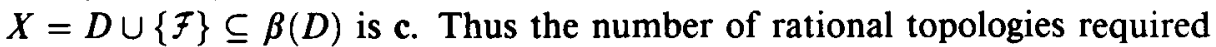
to yield the topology of $X$ is c. This is the most that can ever be required, since the number of metrics on a countable set $S$ is at most $|\mathbf{R}|^{|S \times S|}=\mathbf{c}^{\omega}=\mathbf{c}$.

ReMARK 2.2. By similar methods, one can prove that every completely metrizable zero-dimensional separable space of cardinality $c$ is a join of two irrational topologies. Conversely, a join of two irrational topologies has to be zero-dimensional, metrizable and separable, but it need not be completely metrizable. It would be interesting to characterise topologically the joins of two irrational topologies, and the joins of arbitrary collections of irrational topologies.

The authors are thankful to the referee for his suggestion to include Remarks 1.4 and 2.1. 


\section{References}

[1] W. Sierpiński, 'Sur une propriété topologique des ensembles denombrables dense en soi', Fund. Math. 1 (1920), 11-16.

[2] S. Willard, General topology, (Addison-Wesley, 1970).

School of Mathematics \&

Computer/Information Sciences

University of Hyderabad

Hyderabad-500 134

India 